\title{
HYDROTHERMAL AND MICROWAVE SYNTHESIS OF ZnS NANOPARTICLES
}

\author{
1'Julie SMIJOVÁ, ${ }^{1}$ Pavlína PEIKERTOVÁ, ${ }^{1}$ Kateřina MAMULOVÁ KUTLÁKOVÁ, \\ 1,2,3Jonáš TOKARSKÝ
}

\author{
${ }^{1}$ Nanotechnology Centre, VŠB - Technical University of Ostrava, Ostrava, Czech Republic, EU, \\ julie.smijova.st@vsb.cz, pavlina.peikertova@vsb.cz, katerina.mamulova.kutlakova@vsb.cz, \\ jonas.tokarsky@vsb.cz \\ 2IT4Innovations, VŠB - Technical University of Ostrava, Ostrava, Czech Republic, EU, \\ jonas.tokarsky@vsb.cz \\ ${ }^{3}$ Institute of Environmental Technology, VŠB - Technical University of Ostrava, Ostrava, \\ Czech Republic, EU, jonas.tokarsky@vsb.cz
}

https://doi.org/10.37904/nanocon.2020.3704

\begin{abstract}
Zinc sulfide (ZnS) is a well-known semiconductor with the wide band gap 3.6 eV. Since the ZnS exhibits excellent transmission properties, high electron mobility, non-toxicity, water insolubility, and it is relatively inexpensive, it has been considered as an important photocatalyst for water and wastewater treatment. In this study, ZnS nanoparticles were successfully prepared by hydrothermal synthesis using zinc chloride and sodium sulfide. Nevertheless, in order to further reduce the size of prepared ZnS nanoparticles and thus to further increase their photocatalytic activity, also the microwave synthesis was tested. Phase composition, morphology, particle size, and photoactivity of $\mathrm{ZnS}$ nanoparticles prepared by both methods were compared. FTIR spectroscopy, Raman microspectroscopy, scanning electron microscopy, and X-ray powder diffraction analyses were used. The efficiency of prepared photocatalysts was established by the decomposition of organic azo dye Acid Orange 7 under ultraviolet irradiation.
\end{abstract}

Keywords: ZnS, hydrothermal synthesis, microwave synthesis, photocatalytic activity

\section{INTRODUCTION}

Nanostructured materials have attracted much attention due to their unique properties that are different from bulk materials. Zinc sulfide ( $\mathrm{ZnS})$ is semiconductor with wide band gap ( 3.6 eV) [1]. This is the one of reasons why $\mathrm{ZnS}$ has been used for the light emitting diodes, flat panel displays, electroluminescent, and infrared devices [1]. Nanoparticles ZnS have also a great potential in antibacterial application due to the large surface to volume ratio in comparison with bulk ones. Antibacterial effects of ZnS nanoparticles (2-4 nm) against some pathogen bacteria (Pseudomonas aeruginosa, Actinomycet, Salmonella typhi) have been observed [2]. Various precursors are used for ZnS nanoparticles preparation. For example, zinc acetate [3-5], zinc chloride [6], zinc sulphate [7], or zinc nitrate [6] can be used as a source of zinc ions. Sulfur source can be, for example, thioacetamide [3,4] or thiourea [6]. In this paper, we report a rapid microwave method compared with hydrothermal synthesis for simple preparation of $\mathrm{ZnS}$ nanoparticles synthesized from sodium sulfide $\left(\mathrm{Na}_{2} \mathrm{~S}\right)$ and zinc chloride $\left(\mathrm{ZnCl}_{2}\right)$. Hydrothermal synthesis is a simple one-pot method which does not acquire temperatures above $100^{\circ} \mathrm{C}$. Main principle of microwave synthesis is transferring energy from microwaves to the material, which causes particle reduction. In comparison to hydrothermal synthesis, the microwaveassisted synthesis is much faster, cleaner, energy efficient, and more economical [8]. Preparation process of hydrothermal synthesis was optimized [9], and the microwave synthesis parameters were chosen according 
to previous studies $[3,4,6]$. The precursors were chosen to develop economically acceptable production without by-products and harmful effects on the environment. The aim of this work was to compare the nanostructured material of ZnS prepared by hydrothermal and microwave synthesis. The resulting nanostructured materials were characterized by scanning electron microscopy (SEM), X-ray powder diffraction (XRPD), Fourier transform infrared spectroscopy (FTIR), and Raman microspectroscopy. Photocatalytic activities of both prepared nanostructured materials were compared.

\section{MATERIALS AND METHODS}

A solution of $\mathrm{ZnCl}_{2}(0.5 \mathrm{M})$ and solution of $\mathrm{Na}_{2} \mathrm{~S}(1 \mathrm{M})$ were combined, and later the formed solution was diluted with distilled water. Total volume of the solution was $200 \mathrm{ml}$. The obtained solution was heated in a microwave oven (SENCOR, $700 \mathrm{~W}$ ) operating at $50 \%$. The heating time in the microwave was 30 minutes, the solution was stirred and temperature was measured after every 10 minutes. The same preparation of solution was applied to hydrothermal synthesis. Solution was heated (up to $100{ }^{\circ} \mathrm{C}$ ) and stirred on a magnetic stirrer (Heidolph, MR Hei-Standard) for 3.5 hours. Precipitates were separated from solution by centrifugation and washed repeatedly with water. SEM analysis was performed using JEOL JSM-7610Fplus. Samples were coated by thin layer $(20 \mathrm{~nm})$ of $\mathrm{Pt}$ to make surface conductive, and to prevent charge accumulation. GWYDDION software was used to determine particle sizes. XRPD patterns were recorded (range $5-80^{\circ} 2 \theta$ ) in reflection mode in symmetrical Bragg-Brentano arrangement using Bruker D8 Advance diffractometer equipped with fast position sensitive detector VÅNTEC 1. Radiation CoKa was used $(\lambda=0.1789 \mathrm{~nm})$. Sizes of ZnS crystallites were calculated from the most intensive reflection, i.e. ZnS(111), using Scherrer equation [10].

$L_{c}=\frac{K \cdot \lambda}{\beta \cdot \cos \theta}$

where:

$\mathrm{K}$ - dimensionless shape factor $(0.9)$

$\lambda$ - radiation wavelength $(\mathrm{nm})$

$\beta$ - full width at half maximum (FWHM) of the $\mathrm{ZnS}(111)$ reflection $\left({ }^{\circ}\right)$

$\theta$ - position of the $\mathrm{ZnS}(111)$ reflection $\left({ }^{\circ}\right)$.

FTIR spectra in range $400-4000 \mathrm{~cm}^{-1}$ were recorded by Nicolet 6700 - Thermo Fisher Scientific with diamond ATR crystal (spectral resolution $4 \mathrm{~cm}^{-1}, 32$ scans). The measured data were processed by the OMNIC software. Raman spectra were acquired on Smart Raman System XploRA ${ }^{\mathrm{TM}}$ in range $120-1200 \mathrm{~cm}^{-1}$. Laser $(785 \mathrm{~nm})$ was reduced to $50 \%$ of the initial intensity. Grating with 600 grooves $/ \mathrm{mm}$ and objective with magnification 50x were used. The acquisition time was set to $10 \mathrm{~s}$ with 10 times repetition. Photocatalytic activity (PA) was tested as follows. For each sample, two suspensions containing Acid Orange 7 (AO7) aqueous solution $\left(\mathrm{V}=5 \cdot 10^{-3} \mathrm{dm}^{3}, \mathrm{c}=6.259 \cdot 10^{-4} \mathrm{~mol} / \mathrm{dm}^{3}\right), 50 \mathrm{ml}$ of demineralized water, and $50 \mathrm{mg}$ of the sample were stirred for $1 \mathrm{~h}$ in the dark to obtain the adsorption equilibrium. One suspension was further stirred under UV irradiation $(\lambda=254 \mathrm{~nm})$ for $1 \mathrm{~h}$, the second one (control) was stored in the dark. Discoloration was evaluated using CINTRA 303 UV-VIS spectrometer according to the equation

$P A(\%)=\left(1-\left(\frac{A_{i}}{A_{c}}\right)\right) \cdot 100$

where:

$A_{i}$ - intensity of $A O 7$ absorption maximum ( $480 \mathrm{~nm}$ ) for irradiated suspension

$\mathrm{A}_{c}$ - intensity of $\mathrm{AO} 7$ absorption maximum $(480 \mathrm{~nm})$ for control suspension. 


\section{RESULTS AND DISCUSSION}

\subsection{Scanning electron microscopy}

SEM images of ZnS_H and ZnS_M samples (Figure 1) show high degree of agglomeration. In both samples, the sizes of agglomerates are in micrometer range. However, sizes of the observed particles vary. While agglomerates in ZnS_H sample contain small round shaped (nano)particles having size in range 50 - $200 \mathrm{~nm}$, agglomerates in ZnS_M sample consist of (nano)particles having size $<150 \mathrm{~nm}$. EDS spectra showed the presence of $\mathrm{Zn}, \mathrm{S}$, and $\mathrm{Na}$ elements in both samples, only in the selected spectrum of the ZnS_M sample (shown in Figure 1) Zn band overlaps $\mathrm{Na}$ band. Occurrence of $\mathrm{Pt}$ and $\mathrm{C}$ elements is associated with sample preparation for the SEM analysis. Oxygen may indicate the atmospheric water on the surface of particles.
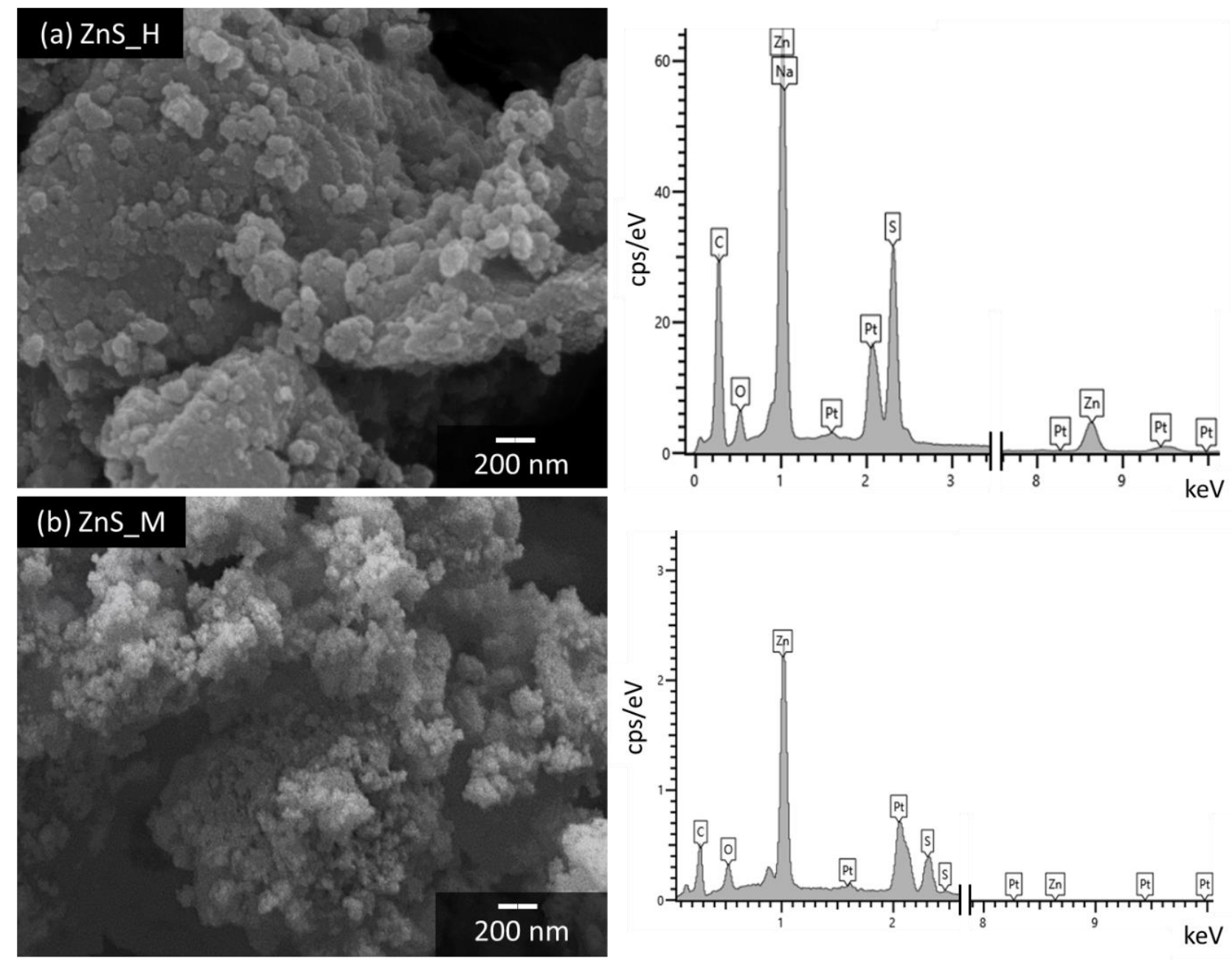

Figure 1 SEM images with corresponding EDS spectra of synthesized ZnS_H (a) and ZnS_M (b) samples

\subsection{Phase analysis}

XRPD analysis (Figure 2) revealed that both ZnS_M and ZnS_H samples contain the same ZnS phase: the cubic phase sphalerite (PDF no. 005-0566). Width of the reflections indicates a low degree of crystallinity. FWHM value for the ZnS_H and ZnS_M sample are $2.894^{\circ}$ and $2.824^{\circ}$, respectively. $L_{c}$ values, calculated according to the equation (1), are similar for both samples, $3.33 \mathrm{~nm}$ (ZnS_H sample) and $3.41 \mathrm{~nm}$ (ZnS_M sample). Apart from ZnS, the XRPD analysis did not show the presence of any other phases. 

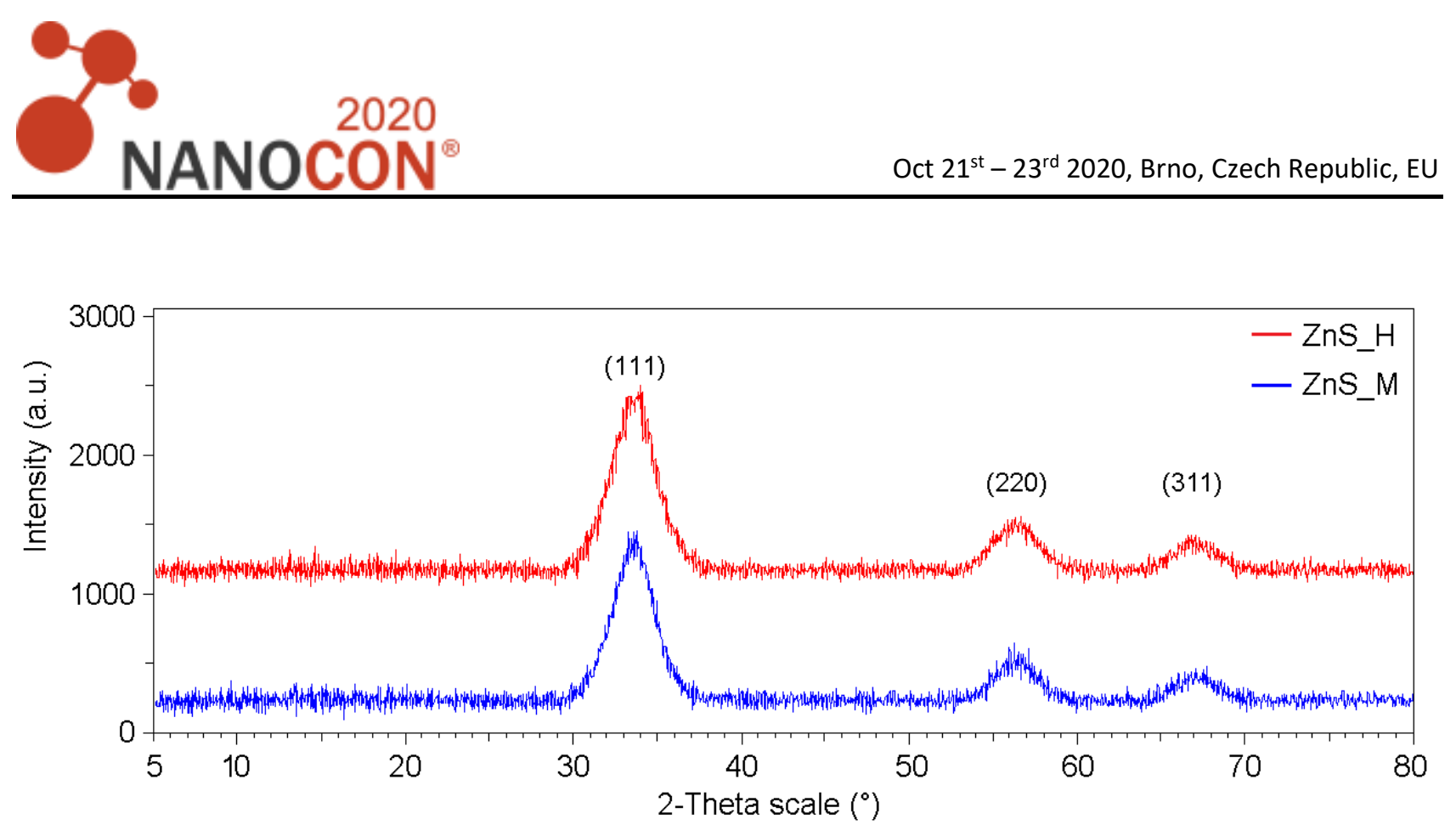

Figure 2 XRPD patterns of synthesized ZnS_M and ZnS_H samples

\subsection{Fourier Transform Infrared Spectroscopy}

Measured FTIR spectra are shown in Figure 3, and it is clearly evident that both samples have similar band position. Thus, the different preparation methods lead to the same composition of the sample, which is in agreement with XRPD results. The most intensive bands at $\sim 3230 \mathrm{~cm}^{-1}$ and $\sim 1629 \mathrm{~cm}^{-1}$ correspond to the stretching and bending vibration of $-\mathrm{OH}$, respectively. Presence of $-\mathrm{OH}$ group indicates the absorbed water on the surface of (nano)crystals originating from atmospheric humidity. Based on the data from literature, the obtained spectra corresponds to the ZnS spectra, and the Zn-S bond can be observed at positions $\sim 1111 \mathrm{~cm}^{-1}, \sim 977 \mathrm{~cm}^{-1}$, and $\sim 635 \mathrm{~cm}^{-1}[11,12]$.

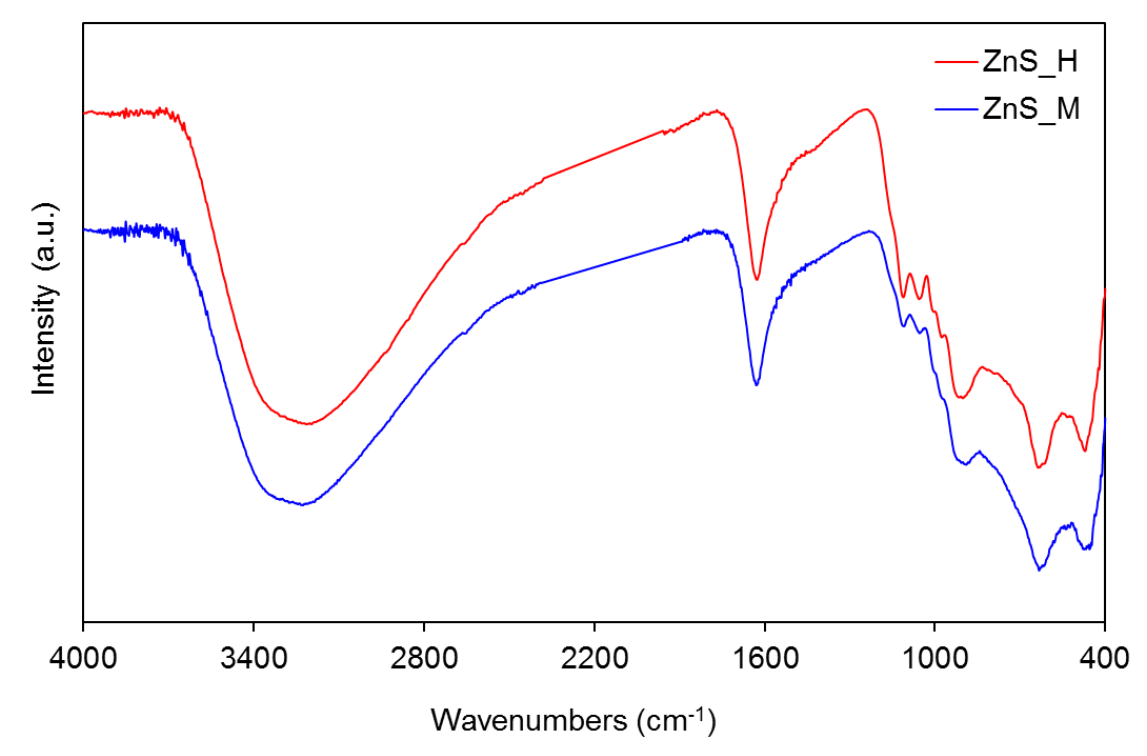

Figure 3 Infrared spectra of synthesized ZnS_M a ZnS_H samples

\subsection{Raman microspectroscopy}

Raman spectra for both ZnS_M and ZnS_H samples are shown in Figure 4. Similarly to the results of XRPD and IR analyses, Raman spectra reveal that both ways of preparation lead to the same ZnS phase as the spectra has similar shape and band positions. According to the bands appearance and bands positions, the ZnS cubic phase, i.e. sphalerite, is confirmed due to the presence of bands at $276 \mathrm{~cm}^{-1}$ and $352 \mathrm{~cm}^{-1}$, which corresponds to the transverse and longitudinal optical zone center phonons of the cubic ZnS sphalerite phase 
[13]. The shape of the Raman spectra (Figure 4) is partially caused by the amorphous-like structure of the ZnS and partially by size of the synthetized particles (in nano range) [14].

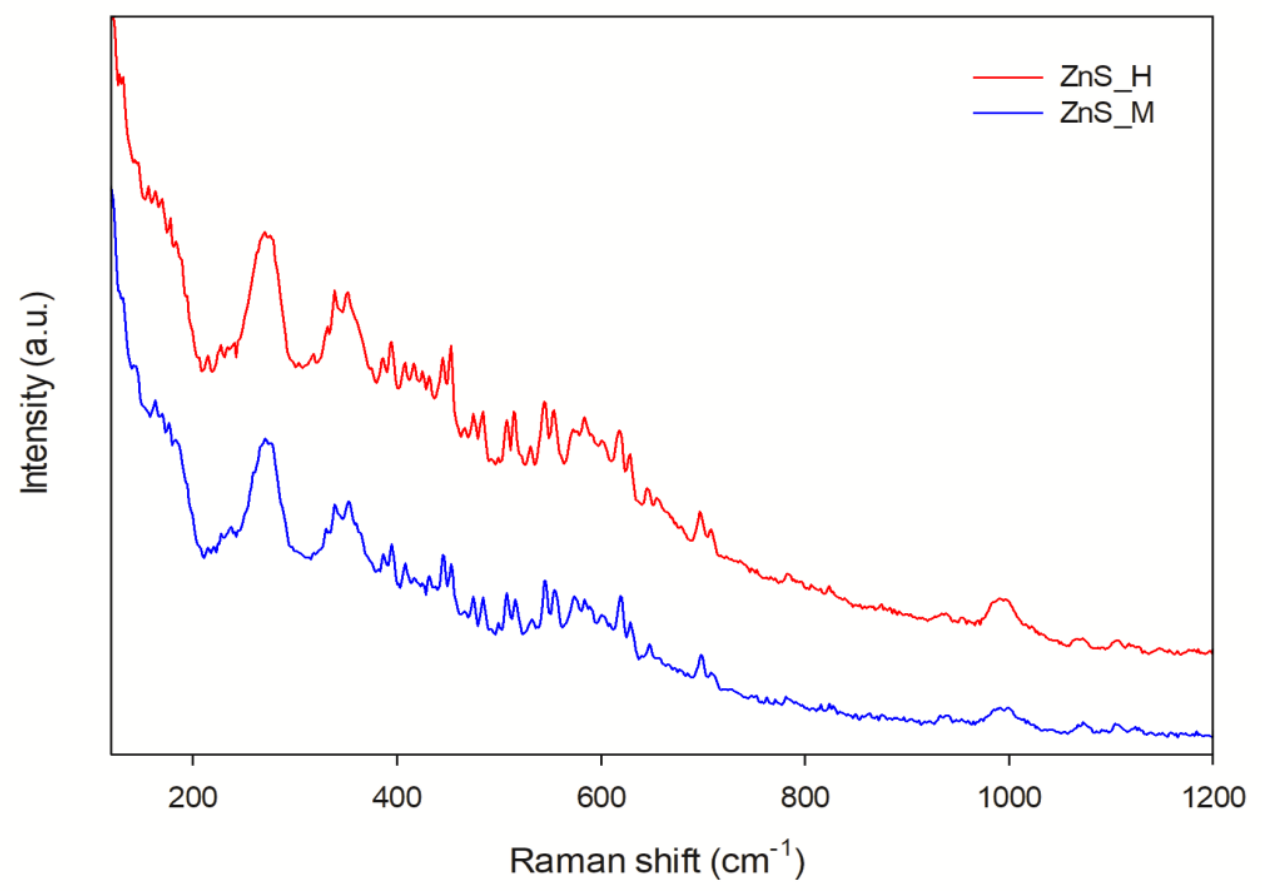

Figure 4 Raman spectra of synthesized ZnS_M a ZnS_H samples.

\subsection{Photocatalytic activity}

ZnS samples prepared by hydrothermal and microwave synthesis do not show a significant difference in photocatalytic activity. Although no change in the color of the AO7 solution was visually observed during the first half of the total duration $(1 \mathrm{~h})$ of the experiment, the resulting PA values for both $\mathrm{ZnS} \_\mathrm{H}$ and $\mathrm{ZnS}$ _M sample were very satisfactory. PA values, calculated according to the equation (2), are $99.27 \%(\mathrm{ZnS} H$ ) and 98.06\% (ZnS_M). The photocatalytic efficiency of both ZnS_H and ZnS_M samples can therefore be considered very good.

\section{CONCLUSION}

Hydrothermal and microwave synthesis led to the successful synthesis of ZnS nanoparticles. The ZnS nanoparticles are not separate; the resulting $\mathrm{ZnS} \_\mathrm{H}$ and $\mathrm{ZnS} \_\mathrm{M}$ samples consist of their nanostructured agglomerates. While SEM analysis showed slight morphological differences between the samples, XRPD, IR, and Raman analyzes consistently confirmed cubic (sphalerite) ZnS phase in both samples, and revealed no significant differences between them. Both samples show high photocatalytic activity. After $1 \mathrm{~h}$ of UV irradiation, more than 99\% (ZnS_H) and more than 98\% (ZnS_M) of the AO7 dye was degraded. The microwave synthesis is faster and more energy efficient than the hydrothermal synthesis. Microwave radiation ensuring uniform heating of the reaction mixture makes the microwave synthesis a promising method for preparing large amount of this highly efficient ZnS photocatalyst.

\section{ACKNOWLEDGEMENTS}

This work was supported by The Ministry of Education, Youth and Sports of the Czech Republic (projects SP2020/24, LQ1602, CZ.02.1.01/0.0/0.0/16_013/0001791). 


\section{REFERENCES}

[1] YAMAMOTO, T., KISHIMOTO, S., IIDA, S. Control of valence states for ZnS by triple-codoping method. Physica B: Condensed Matter. 2001, vol. 308-310, pp. 916-919.

[2] AMIR, G.R., FATAHIAN, S., KIANPOUR, N. Investigation of ZnS Nanoparticle Antibacterial Effect. Current Nanoscience. 2014, vol. 10, no. 6, pp. 796-800.

[3] SOLTANI, N., SAION, E., YUNUS, W.M.M., ERFANI, M., NAVASERY, M., BAHMANROKH, G., REZAEE, K. Enhancement of visible light photocatalytic activity of ZnS and CdS nanoparticles based on organic and inorganic coating. Applied Surface Science. 2014, vol. 290, pp. 440-447.

[4] RATH, T., KUNERT, B., RESEL, R., FRITZ-POPOVSKI, G., SAF, R., TRIMMEL, G. Investigation of Primary Crystallite Sizes in Nanocrystalline ZnS Powders: Comparison of Microwave Assisted with Conventional Synthesis Routes. Inorganic Chemistry. 2008, vol. 47, no. 8, pp. 3014-3022.

[5] GOHARSHADI, E.K., MEHRKHAH, R., NANCARROW, P. Synthesis, characterization, and measurement of structural, optical, and phtotoluminescent properties of zinc sulfide quantum dots. Materials Science in Semiconductor Processing. 2013, vol. 16, no. 2, pp. 356-362.

[6] LA PORTA, F.A., FERRER, M.M., DE SANTANA, Y.V.B., RAUBACH, C.W., LONGO, V.M., SAMBRANO, J.R., LONGO, E., ANDRÉS, J., LI, M.S., VARELA, J.A. Synthesis of wurtzite ZnS nanoparticles using the microwave assisted solvothermal method. Journal of Alloys and Compounds. 2013, vol. 556, pp. 153-159.

[7] VÁZQUEZ, A. a J. AGUILAR-GARIB. Preparation of ZnS nanoparticles using microwave assisted synthesis: effects of the irradiation power and the precursors. Revista Mexicana de Física. Mexico: Mayo, 2009, vol. S55, no. 1, pp. 57-60.

[8] RAO, J.K., VAIDHYANATHAN, B., GANGUL, M., RAMAKRISHNAN, P.A. Synthesis of Inorganic Solids Using Microwaves. Chemistry Of Materials. 1999, vol. 11, no. 4, pp. 882-895.

[9] MAMULOVÁ KUTLÁKOVÁ, K., TOKARSKÝ, J. Simple one-pot hydrothermal synthesis and photocatalytic activity of ZnS and ZnS/kaolinite nanocomposite. In: NANOCON 2019: 1th International Conference on Nanomaterials Research \& Application. Ostrava: TANGER, 2019, pp. 146-151.

[10] SCHERRER, P. Estimation of size and internal structure of colloidal particles by means of Röntgen rays. Nachrichten von der Gesellschaft der Wissenschaften zu Göttingen. 1918, vol. 2, pp. 96-100.

[11] IRANMANESH, P., SAEEDNIA, S., NOURZPOOR, M. Characterization of ZnS nanoparticles synthesized by coprecipitation method. Chinese Physics B. 2015, vol. 24, no. 4.

[12] MOTE, V.D., PURUSHOTHAM, Y., DOLE, B.N. Structural, morphological and optical properties of Mn doped ZnS nanocrystals. Cerâmica. 2013, vol. 59, no. 351, pp. 395-400.

[13] KUMAR, S.S., KHADAR, M.K., DHARA, S.K., RAVINDRAN, T.R., NAIR, K.G.M. Photoluminescence and Raman studies of ZnS nanoparticles implanted with $\mathrm{Cu}^{+}$ions. Nuclear Instruments and Methods in Physics Research Section B: Beam Interactions with Materials and Atoms. 2006, vol. 251, no. 2, pp. 435-440.

[14] ABDULKHADAR, M., THOMAS, B. Study of Raman spectra of nanoparticles of CdS and ZnS. NanoStructured Materials. 1995, vol. 5, no. 3, pp. 289-298. 